\title{
Measurement of high-energy prompt gamma-rays from neutron induced fission of U-235
}

\author{
Hiroyuki Makii ${ }^{1, a}$, Katsuhisa Nishio ${ }^{1}$, Kentaro Hirose ${ }^{1}$, Riccardo Orlandi ${ }^{1}$, Romain Léguillon ${ }^{1}$, Tatsuhiko Ogawa ${ }^{2}$, \\ Torsten Soldner ${ }^{3}$, Franz-Josef Hambsch ${ }^{4}$, Alain Astier ${ }^{5}$, Andrew Pollitt ${ }^{3}$, Costel Petrache ${ }^{5}$, Igor Tsekhanovich ${ }^{6}$, \\ Ludovic Mathieu ${ }^{6}$, Mourad Aïche $^{6}$, Robert Frost ${ }^{7}$, Serge Czajkowski ${ }^{6}$, Song Guo ${ }^{5}$, and Ulli Köster ${ }^{3}$ \\ 1 Advanced Science Research Center, Japan Atomic Energy Agency, Tokai, Ibaraki 319-1195, Japan \\ 2 Nuclear Science and Engineering Center, Japan Atomic Energy Agency, Tokai, Ibaraki 319-1195, Japan \\ 3 Institut Laue-Langevin, 38000 Grenoble, France \\ ${ }^{4}$ European Commission, Joint Research Centre - IRMM, 2440 Geel, Belgium \\ 5 Centre de Sciences Nucléaires et de Sciences de la Matière, Université Paris-Sud and CNRS/IN2P3, 91405 Orsay, France \\ ${ }^{6}$ Centre Etudes Nucléaires de Bordeaux Gradignan, 33175 Gradignan Cedex, France \\ 7 School of Physics and Astronomy, University of Manchester, M13 9PL Manchester, UK
}

\begin{abstract}
We have developed a new setup to measure prompt $\gamma$-rays from the ${ }^{235} \mathrm{U}\left(n_{t h}, f\right)$ reaction. The setup consists of two multi-wire proportional counters (MWPCs) to detect the fission fragments, two $\mathrm{LaBr}_{3}(\mathrm{Ce})$ scintillators to measure the $\gamma$-rays. The highly efficient setup was installed at the PF1B beam line of the Institut Laue Langevin (ILL). We have successfully measured the $\gamma$-ray spectrum up to about $20 \mathrm{MeV}$ for the fist time in neutron-induced fission.
\end{abstract}

\section{Introduction}

The measurement of the prompt $\gamma$-ray spectrum (PFGS) is quite important to study the de-excitation process of neutron-rich fission fragments [1] as well as to generate data required to design new types of reactors, such as the Generation-IV reactors and advanced lightwater reactors (Generation-III ${ }^{+}$reactors) [2]. The PFGS measured for spontaneous fission of ${ }^{252} \mathrm{Cf}[3,4]$ does not decrease linearly with $\gamma$-ray energy on a logarithmic scale but shows a broad hump at $\gamma$-ray energies of $E_{\gamma} \geq$ $8 \mathrm{MeV}$. This is interpreted as a GDR of fission fragments centered around $E_{\gamma}=15 \mathrm{MeV}$. In addition to a study of the GDR for neutron-rich nuclei, the PFGS includes information of sharing the total excitation energy between two fragments [5], and information on the structure of neutron-rich nuclei [6] which plays a key role in deexcitation process of the highly excited fragments. The measurement of the PFGS is also connected to the decommissioning of the nuclear-fuel debris in damaged Fukushima atomic power plants in Japan. This necessitates a surveillance detector to monitor the fission rate in the debris. The prompt $\gamma$-rays in fission with the energies higher than $E_{\gamma}=6 \mathrm{MeV}$, which corresponds to the highest energy of background $\gamma$-rays from the debris, would be used to monitor the fission rate. For thermal neutron-induced fission of ${ }^{235} \mathrm{U},{ }^{235} \mathrm{U}\left(n_{t h}, f\right)$, the data of the PFGS are limited up to $E_{\gamma} \sim 8 \mathrm{MeV}$ [7] and $E_{\gamma} \sim 6 \mathrm{MeV}$ [8], respectively. This prompted us to make a new measurement to extend the known PFGS up to energies high enough to observe the GDR for the ${ }^{235} \mathrm{U}\left(n_{t h}, f\right)$ reaction using the newly developed

a e-mail: makii.hiroyuki@jaea.go.jp multi-wire proportional counters (MWPCs) and $\mathrm{LaBr}_{3}(\mathrm{Ce})$ scintillators. A digital data acquisition system was used to acquire signals from the detectors with high counting rate and to monitor pile-up events. By using the setup, we have measured the PFGS for the ${ }^{235} \mathrm{U}\left(n_{t h}, f\right)$ reaction at the Institut Laue Langevin (ILL), Grenoble, France. The experimental method and results are presented in this work.

\section{Experiment}

The measurement was carried out at the PF1B beam line of the ILL [9]. In the present study, we have used two MWPCs to detect both fission fragments (FFs) in coincidence and two $\mathrm{LaBr}_{3}(\mathrm{Ce})$ scintillators to measure $\gamma$-rays accompanying the ${ }^{235} \mathrm{U}\left(n_{t h}, f\right)$ reaction. The experimental setup is shown in Fig. 1.

A ${ }^{235} \mathrm{U}$ target (isotopic enrichment is 99.9\%), $117 \mu \mathrm{g} / \mathrm{cm}^{2}$ in thickness and $30 \mathrm{~mm}$ in diameter, evaporated on $33 \mu \mathrm{g} / \mathrm{cm}^{2}$ polyimide backing, was mounted with a tilting angle of $45^{\circ}$ with respect to the beam axis in the vacuum chamber made of $\mathrm{Al}$ as shown in Fig. 1. The neutron beam was collimated to about $20 \mathrm{~mm}$ in diameter to give a neutron flux of $1.0 \times 10^{8} \mathrm{n} / \mathrm{cm}^{2} / \mathrm{s}$ at the target position to optimize a counting rate of the MWPCs and the $\mathrm{LaBr}_{3}(\mathrm{Ce})$ scintillators. The counting rates for the MWPCs and $\mathrm{LaBr}_{3}(\mathrm{Ce})$ scintillators were about $55 \mathrm{kHz}$ and $30 \mathrm{kHz}$, respectively. The neutron beam passed through the thin aluminum windows to irradiate ${ }^{235} \mathrm{U}$ target mounted at the center of the vacuum chamber.

Both FFs were detected by $80 \times 80 \mathrm{~mm}^{2}$ positionsensitive MWPCs attached in the vacuum chamber. The distance between the target-center and the middle of the MWPC cathode was $50 \mathrm{~mm}$ corresponding to a geometric

(C) The Authors, published by EDP Sciences. This is an Open Access article distributed under the terms of the Creative Commons Attribution License 4.0 (http://creativecommons.org/licenses/by/4.0/). 


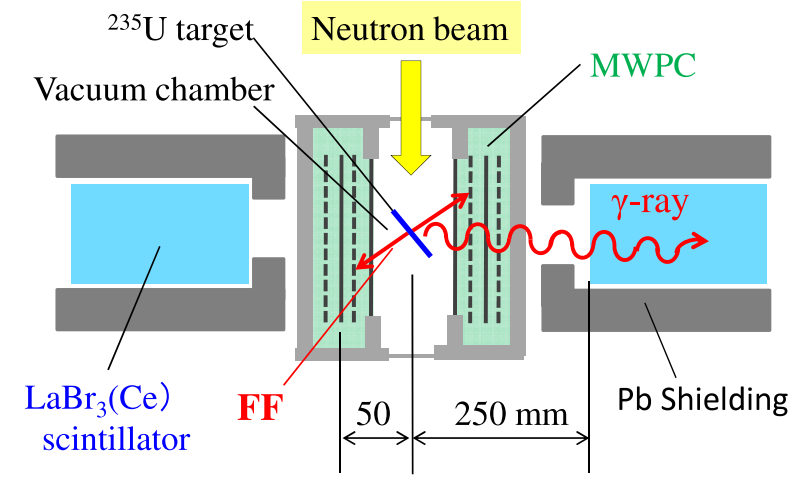

Figure 1. Schematic view of the experimental setup.

efficiency of $30 \%$. The MWPCs were operated with tetrafluoromethane gas at a pressure of 4.5 Torr. A $3 \mu \mathrm{m}$ aluminum-coated Mylar film was used as an entrance window. The positions of FFs's incidence on the MWPC were determined with a position resolution of $2.0 \mathrm{~mm}$.

Two $\mathrm{LaBr}_{3}(\mathrm{Ce})$ scintillators with large volume (101.6 $\mathrm{mm}$ in diameter and $127 \mathrm{~mm}$ in length) were used to detect the $\gamma$-rays from the fragments. The distance between the center of target and the front face of the $\mathrm{LaBr}_{3}(\mathrm{Ce})$ scintillator was set to be $250 \mathrm{~mm}$ to separate a prompt $\gamma$-rays and neutrons with a time-offlight (TOF) method as will be described below. The $\mathrm{LaBr}_{3}(\mathrm{Ce})$ scintillators have a detection efficiency of $0.7 \%$ for $1.33 \mathrm{MeV} \gamma$-ray.

Signals from the MWPCs and $\mathrm{LaBr}_{3}(\mathrm{Ce})$ scintillators with their time-stamp information were acquired by a newly developed high-speed digital data acquisition system and recorded on a hard disk. Therefore the coincidence between the MWPCs and $\mathrm{LaBr}_{3}(\mathrm{Ce})$ scintillators can be performed in the off-line analysis. The dead time of the system was less than a few percents during the measurement, allowing almost dead time free measurements. Through the run for 437 hours, the totally registered FFs in coincidence reached $4.3 \times 10^{10}$.

\section{Results}

$\gamma$-ray spectrum obtained by one of the $\operatorname{LaBr}_{3}(\mathrm{Ce})$ scintillators is shown in Fig. 2(a). Here the spectrum was obtained without imposing any gate conditions and the threshold for the $\mathrm{LaBr}_{3}(\mathrm{Ce})$ scintillators was set at $0.8 \mathrm{MeV}$. We see clearly several discrete background $\gamma$-rays up to about $2300 \mathrm{ch}$. They were produced by the collision of neutrons with various materials including the $\operatorname{LaBr}_{3}(\mathrm{Ce})$ scintillator itself. Since Al was used for the vacuum chamber, the housing of the MWPCs and that of the $\mathrm{LaBr}_{3}(\mathrm{Ce})$ scintillators, most of these $\gamma$-rays are explained as the events due to the ${ }^{27} \mathrm{Al}(n, \gamma)^{28} \mathrm{Al}$ reaction and $\beta$-decay of ${ }^{28} \mathrm{Al}$ followed by the neutron capture reaction of ${ }^{27} \mathrm{Al}$, e.g., peaks at around $430 \mathrm{ch}$ and $1850 \mathrm{ch}$ were assigned to be $1.78 \mathrm{MeV} \gamma$-ray from the $\beta$-decay of ${ }^{28} \mathrm{Al}$ and $7.72 \mathrm{MeV} \gamma$-ray from the ${ }^{27} \mathrm{Al}(n, \gamma){ }^{28} \mathrm{Al}$ reaction, respectively. These $\gamma$-rays were used for checking the gain stability of the $\mathrm{LaBr}_{3}(\mathrm{Ce})$ scintillators during the measurement.

Figure 2(b) shows time difference $(\Delta t)$ between FFs and $\gamma$-rays. The correction for time-shift of FFs traversing the $50 \mathrm{~mm}$ distance has been applied by using the positions
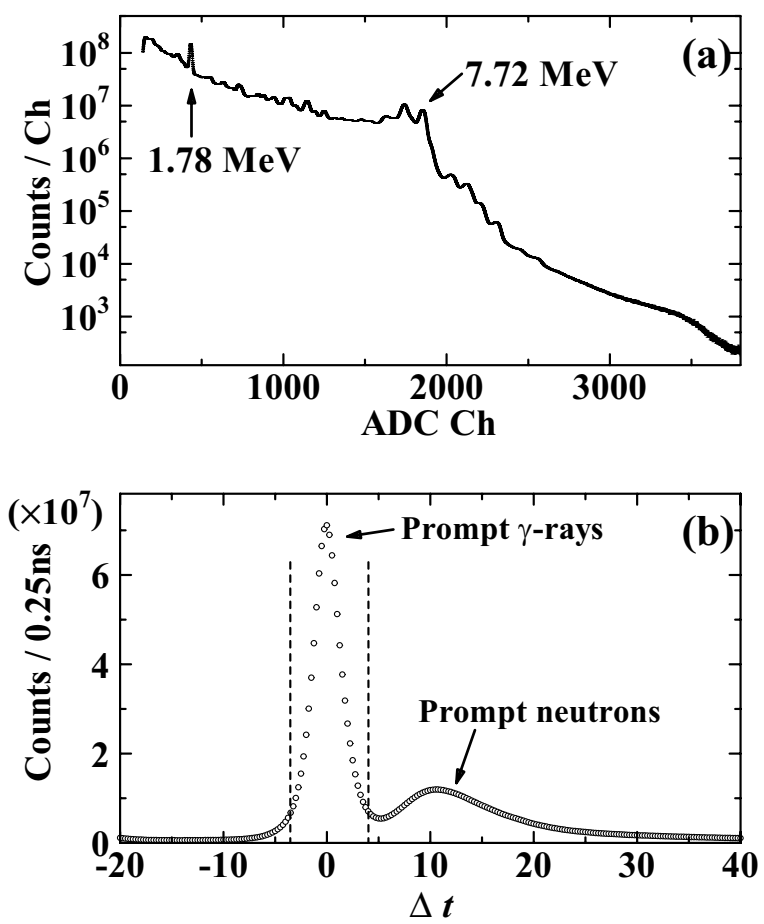

Figure 2. (a) A $\gamma$-ray spectrum taken by the $\operatorname{LaBr}_{3}(\mathrm{Ce})$ scintillator. The spectrum was obtained without imposing any gate conditions. (b) Time difference $(\Delta t)$ between the FFs and $\gamma$-rays. Dashed lines indicate the example of gate position (for ADC ch $\leq 1000 \mathrm{ch}$ ), which was used to obtain the $\gamma$-ray spectrum coincidence with FFs shown below.

of FFs's incidence on the MWPC. A peak at $\Delta t=0 \mathrm{~ns}$ contained $\gamma$-ray events accompanying the ${ }^{235} \mathrm{U}\left(n_{t h}, f\right)$ reaction. The events at a broad peak at $\Delta t \sim 10 \mathrm{~ns}$ were assigned to be background events originating from prompt-neutrons in the ${ }^{235} \mathrm{U}\left(n_{t h}, f\right)$ reaction, the $\left(n, n^{\prime} \gamma\right)$ and/or $(n, \gamma)$ events produced by the interaction with bromine $\left({ }^{79,81} \mathrm{Br}\right)$ and lanthanum $\left({ }^{139} \mathrm{La}\right)$ in the $\mathrm{LaBr}_{3}(\mathrm{Ce})$ scintillator. Since the interaction of neutrons with $\mathrm{LaBr}_{3}(\mathrm{Ce})$ scintillator was well studied in Ref. [10], the events contained in the broad peak would be used to obtain information on prompt-neutrons in the ${ }^{235} \mathrm{U}\left(n_{t h}, f\right)$ reaction in further analysis. The plateau region $(\Delta t \leq$ $-10 \mathrm{~ns})$ is due to time-independent background caused by natural radioactivity including the self-activity of the $\mathrm{LaBr}_{3}(\mathrm{Ce})$ scintillator, thermal neutrons scattered from various materials the neutron beam passed through, and cosmic rays. The time-independent background events were assumed to be contained in the peak at $\Delta t=0 \mathrm{~ns}$ with the same intensity, which was thus subtracted from the $\gamma$-ray spectrum measured in the peak, as will be described below.

The $\gamma$-ray spectrum in coincidence with FFs (foreground spectrum) is indicated by solid line in Fig. 3. The spectrum was obtained by applying the time window for the $\Delta t$, which is indicated by the dashed lines in Fig. 2(b). Here the width of the time window corresponds to full width at tenth maximum of the peak at $\Delta t=0 \mathrm{~ns}$. For high-energy region (ADC ch $>1000$ ), the $\Delta t$ spectrum obtained in the present measurement depended on $\gamma$-ray energy observed by the $\operatorname{LaBr}_{3}(\mathrm{Ce})$ scintillator. Therefore, we adjusted a gate position on the positive side on $\Delta t$ spectrum so as not to include 


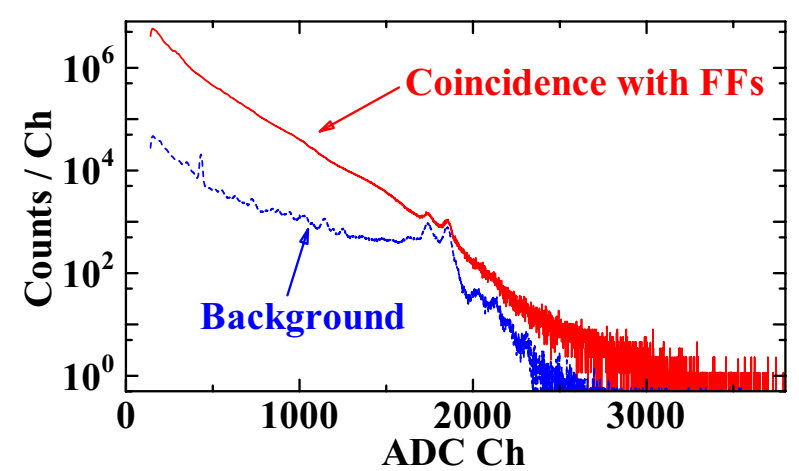

Figure 3. $\gamma$-ray spectrum coincidence with FFs (solid line) compared to time-independent background $\gamma$-ray spectrum (dotted line).

the time-dependent background events observed in the broad peak at $\Delta t \sim 10 \mathrm{~ns}$. Details of the analysis will be published elsewhere[11]. We can see the $7.72 \mathrm{MeV}$ $\gamma$-ray from the ${ }^{27} \mathrm{Al}(n, \gamma)^{28} \mathrm{Al}$ reaction. This indicates that the foreground spectrum contains non-negligible amount of time-independent events. The time-independent background $\gamma$-ray spectrum was obtained by putting the gate on $\Delta t$ spectrum at $\Delta t \leq-10 \mathrm{~ns}$ (see Fig 2(b)), and the resulting spectrum expected to be contained in the peak at $\Delta t=0 \mathrm{~ns}$ is indicated by dotted line in Fig. 3. The background subtracted (net) spectrum was thus obtained by subtracting the time-independent background $\gamma$-ray spectrum from the foreground spectrum.

The PFGS for the ${ }^{235} \mathrm{U}\left(n_{t h}, f\right)$ reaction was deduced by unfolding the net-spectrum with the use of response functions of the $\operatorname{LaBr}_{3}(\mathrm{Ce})$ scintillators. Response functions and also energy calibration of the $\mathrm{LaBr}_{3}(\mathrm{Ce})$ scintillators were made using the $1.173-$ and $1.333-\mathrm{MeV}$ $\gamma$-rays from the ${ }^{60} \mathrm{Co} \gamma$-ray source, and 4.44-, 8.73-, 13.9-, and $18.4-\mathrm{MeV}$ ones from the ${ }^{11} \mathrm{~B}(p, \gamma)^{12} \mathrm{C}$ reaction [12]. The response functions of the scintillators were calculated by using the Monte-Carlo code, GEANT4 [13] in a similar manner described in Ref. [14]. In the present study, the unfolding was performed with a computer program, TUnfold [15], and the response matrix made of response functions mentioned above. Validity of the response functions used in the analysis was confirmed by comparing the calculated spectrum with the $\gamma$-ray spectrum from the ${ }^{11} \mathrm{~B}(p, \gamma){ }^{12} \mathrm{C}$ reaction measured with the same $\operatorname{LaBr}_{3}(\mathrm{Ce})$ scintillators used in this study, as shown in Fig. 4. The preliminary result of the unfolded $\gamma$-ray spectrum for the ${ }^{235} \mathrm{U}\left(n_{t h}, f\right)$ reaction is shown in Fig. 5.

As shown in Fig. 5, we have succeeded to extend the PFGS for the ${ }^{235} \mathrm{U}\left(n_{t h}, f\right)$ reaction up to $E_{\gamma} \sim 20 \mathrm{MeV}$, by gaining the factor of $10^{5}$ in statistics compared to the latest measurement [8], which reports the PFGS up to $E_{\gamma} \sim 6 \mathrm{MeV}$. It is evident that the obtained PFGS for the ${ }^{235} \mathrm{U}\left(n_{t h}, f\right)$ reaction does not decrease linearly with $\gamma$-ray energy on the logarithmic scale, but shows a broad hump at $E_{\gamma} \geq 10 \mathrm{MeV}$. This could be interpreted as the GDR of FFs as with the case of spontaneous fission of ${ }^{252} \mathrm{Cf}$. We have observed the GDR of FFs for the first time in neutroninduced fission. In addition to the hump at $E_{\gamma} \geq 10 \mathrm{MeV}$, hump structures around $E_{\gamma}=4 \mathrm{MeV}$ and $6 \mathrm{MeV}$ are found in Fig. 5. These structures were also found in the PFGS for

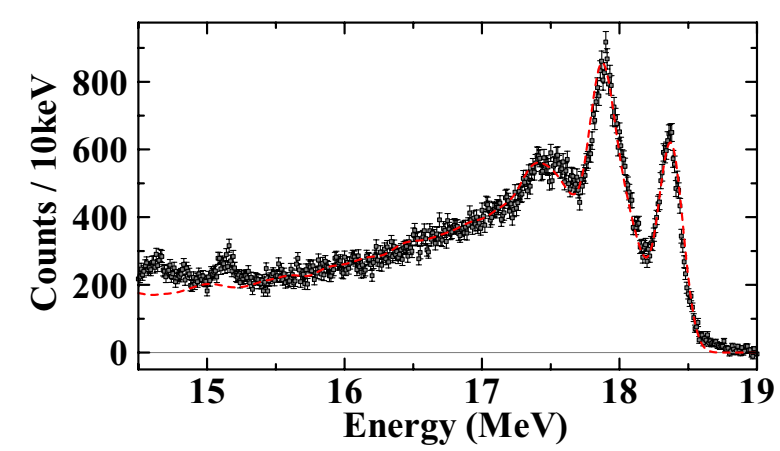

Figure 4. Measured $18.4 \mathrm{MeV} \quad \gamma$-ray spectrum of the ${ }^{11} \mathrm{~B}(p, \gamma){ }^{12} \mathrm{C}$ reaction (black square) compared to the calculated one (dotted line) by GEANT4. The calculated spectrum deviates from the measured one below about $15.3 \mathrm{MeV}$ due to the 15.1 MeV $\gamma$ ray from the same reaction.

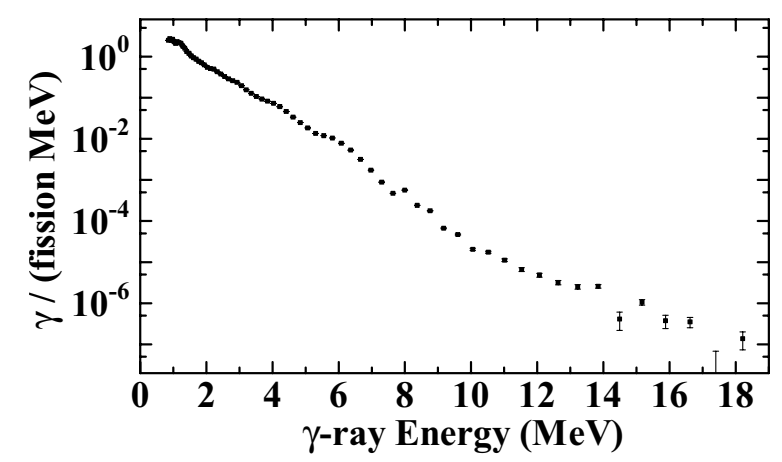

Figure 5. PFGS for the ${ }^{235} \mathrm{U}\left(n_{t h}, f\right)$ reaction (preliminary).

spontaneous fission of ${ }^{252} \mathrm{Cf}$ [16]. The origins of the hump structures in the present PFGS for the ${ }^{235} \mathrm{U}\left(n_{t h}, f\right)$ reaction will be discussed in further publication.

\section{Conclusions}

In this study, we have developed a new setup to measure the prompt $\gamma$-rays in fission with significantly high sensitivity, particularly for the high-energy region. The setup consists of two MWPCs to detect FFs, two large volume $\mathrm{LaBr}_{3}(\mathrm{Ce})$ scintillators. The set up was used to measure the PFGS for the ${ }^{235} \mathrm{U}\left(n_{t h}, f\right)$ reaction using high intense neutron beam available at the PF1B beam line of the ILL. The obtained spectrum reached up to $E_{\gamma} \sim 20 \mathrm{MeV}$, where the structure associated with the GDR was revealed for the first time in neutron-induced fission. We also observed hump structures at around $E_{\gamma}=4 \mathrm{MeV}$ and $6 \mathrm{MeV}$, similar to the observation in spontaneous fission of ${ }^{252} \mathrm{Cf}$. Further measurements are necessary to elucidate the origin of these structures, by making correlation measurement between PFGS and fission fragment masses and/or total kinetic energies. Existence of high energy $\gamma$-rays of $E_{\gamma} \geq 6 \mathrm{MeV}$ and its spectrum could open a way to develop a new type of surveillance detector to monitor the increasing fission rate in the debris of damaged Fukushima atomic power plants that could happen in removing the debris as a first action for the decommissioning.

The present study is the result of "Measurement of high-energy prompt gamma-rays in fission for surveillance detector to monitor 
criticality of fuel debris" entrusted to Japan Atomic Energy Agency (JAEA) by the Ministry of Education, Culture, Sports, Science and Technology of Japan (MEXT).

\section{References}

[1] R. Vandenbosch, J. Huizenga, Nuclear Fission (Academic Press, 1973)

[2] G. Rimpault, D. Bernard, D. Blanchet, C. VaglioGaudard, S. Ravaux, A. Santamarina, Physics Procedia 31, 3 (2012)

[3] H. van der Ploeg, J.C.S. Bacelar, A. Buda, C.R. Laurens, A. van der Woude, J.J. Gaardho/je, Z. Żelazny, G. van 't Hof, N. Kalantar-Nayestanaki, Phys. Rev. C 52, 1915 (1995)

[4] D. Pandit, S. Mukhopadhyay, S. Bhattacharya, S. Pal, A. De, S. Banerjee, Physics Letters B 690(5), 473 (2010)

[5] K. Nishio, Y. Nakagome, H. Yamamoto, I. Kimura, Nuclear Physics A 632(4), 540 (1998)

[6] I. Ahmad, W.R. Phillips, Reports on Progress in Physics 58(11), 1415 (1995)

[7] V.V. Verbinski, H. Weber, R.E. Sund, Phys. Rev. C 7, 1173 (1973)

[8] A. Oberstedt, T. Belgya, R. Billnert, R. Borcea, T. Bryś, W. Geerts, A. Göök, F.J. Hambsch, Z. Kis, T. Martinez et al., Phys. Rev. C 87, 051602 (2013)

[9] H. Abele, D. Dubbers, H. Häse, M. Klein, A. Knöpfler, M. Kreuz, T. Lauer, B. Mrkisch, D. Mund, V. Nesvizhevsky et al., Nuclear Instruments and Methods in Physics Research
Section A: Accelerators, Spectrometers, Detectors and Associated Equipment 562(1), 407 (2006)

[10] A. Oberstedt, R. Billnert, S. Oberstedt, Nuclear Instruments and Methods in Physics Research Section A: Accelerators, Spectrometers, Detectors and Associated Equipment 708, 7 (2013)

[11] H. Makii, K. Nishio, K. Hirose, R. Orlandi, R. Léguillon, T. Ogawa, T. Soldner, F.J. Hambsch, A. Aïche, A. Astier et al., in preparation

[12] F. Zijderhand, F. Jansen, C. Alderliesten, C. van der Leun, Nuclear Instruments and Methods in Physics Research Section A: Accelerators, Spectrometers, Detectors and Associated Equipment 286(3), 490 (1990)

[13] S. Agostinelli, J. Allison, K. Amako, J. Apostolakis, H. Araujo, P. Arce, M. Asai, D. Axen, S. Banerjee, G. Barrand et al., Nuclear Instruments and Methods in Physics Research Section A: Accelerators, Spectrometers, Detectors and Associated Equipment 506(3), 250 (2003)

[14] H. Makii, S. Ota, T. Ishii, Y. Wakabayashi, K. Furutaka, K. Nishio, I. Nishinaka, S. Chiba, M. Igashira, A. Czeszumska, Nuclear Instruments and Methods in Physics Research Section A: Accelerators, Spectrometers, Detectors and Associated Equipment 797, 83 (2015)

[15] S. Schmitt, Journal of Instrumentation 7(10), T10003 (2012)

[16] A. Hotzel, P. Thirolf, C. Ender, D. Schwalm, M. Mutterer, P. Singer, M. Klemens, J.P. Theobald, M. Hesse, F. Gönnenwein et al., Zeitschrift für Physik A Hadrons and Nuclei 356(3), 299 (1996) 